TITLE:

\title{
Dose-dependent functionality and toxicity of green tea polyphenols in experimental rodents.
}

$\operatorname{AUTHOR}(S)$ :

Murakami, Akira

CITATION:

Murakami, Akira. Dose-dependent functionality and toxicity of green tea polyphenols in experimental rodents.. Archives of biochemistry and biophysics 2014, 557: 3-10

ISSUE DATE:

2014-09-01

URL:

http://hdl.handle.net/2433/189425

RIGHT:

(c) 2014 Elsevier Inc.; この論文は出版社版でありません。引用の際には 出版社版をご確認ご利用ください。; This is not the published version. Please cite only the published version. 
3

4

Akira Murakami

5

6

Division of Food Science and Biotechnology

7

Graduate School of Agriculture

8

Kyoto University, Kyoto, 606-8502, Japan

9

TEL: +81-75-753-6282; FAX: +81-75-753-6284; Email: cancer@kais.kyoto-u.ac.jp

10

11

12 


\section{Abstract}

2 A large number of physiologically functional foods are comprised of plant polyphenols.

3 Their antioxidative activities have been intensively studied for a long period and proposed to

4 be one of the major mechanisms of action accounting for their health promotional and

5 disease preventive effects. Green tea polyphenols (GTPs) are considered to possess marked

6 anti-oxidative properties and versatile beneficial functions, including anti-inflammation and

7 cancer prevention. On the other hand, some investigators, including us, have uncovered their

8 toxicity at high doses presumably due to pro-oxidative properties. For instance, both

9 experimental animal studies and epidemiological surveys have demonstrated that GTPs may

10 cause hepatotoxicity. We also recently showed that diets containing high doses (0.5-1\%) of a

11 GTP deteriorated dextran sodium sulfate (DSS)-induced intestinal inflammation and

12 carcinogenesis. In addition, colitis mode mice fed a 1\% GTP exhibited symptoms of

13 nephrotoxicity, as indicated by marked elevation of serum creatinine level. This diet also

14 increased thiobarbituric acid-reactive substances, a reliable marker of oxidative damage, in

15 both kidneys and livers even in normal mice, while the expression levels of antioxidant

16 enzymes and heat shock proteins (HSPs) were diminished in colitis and normal mice.

17 Intriguingly, GTPs at $0.01 \%$ and $0.1 \%$ showed hepato-protective activities, i.e., they

18 significantly suppressed DSS-increased serum aspartate aminotransferase and alanine 
1 aminotransferase levels. Moreover, those diets remarkably restored DSS-down-regulated

2 expressions of heme oxygenase-1 and HSP70 in livers and kidneys. Taken together, while

3 low and medium doses of GTPs are beneficial in colitis model mice, unwanted side-effects

4 occasionally emerge with high doses. This dose-dependent functionality and toxicity of

5 GTPs are in accordance with the concept of hormesis, in which mild, but not severe, stress

6 activates defense systems for adaptation and survival.

7

8 Keywords: green tea polyphenol; colitis; nephrotoxicity; heat shock protein; molecular

9 chaperone; pro-oxidation

10

11 Abbreviations: ABC, ATP-binding cassette; ACR, acrolein; ALS, amyotrophic lateral

12 sclerosis; ALT, alanine aminotransferase; AST, aspartate aminotransferase: DMH,

13 dimethylhydrazine; DSS, dextran sulfate sodium; EGCG, (-)-epigallocatehin-3-gallate; ERK,

14 extracellular signal-regulated kinase; GTP, green tea polyphenols; GSH, glutathione; GST,

15 GSH S-transferase; HNE, 4-hydroxy-2-nonenal; HO-1, heme oxygenase-1; Hsf, heat shock

16 factor; HS, heat shock; HSP, heat shock protein; Keap1, Kelch-like ECH-associated protein;

17 MDA, malondialdehyde; MMP, matrix metalloproteinase; Nrf2, nuclear factor

18 (erythroid-derived 2)-like 2; NQO1, NAD(P)H dehydrogenase; PQC, protein quality control; 
1 ROS, reactive oxygen species; SOD, superoxide dismutase; TBARS, thiobarbituric

2 acid-reactive substances; Ub, ubiquitin

\section{Stress-induced biomolecule modifications}

5 membrane lipids, enzymes, proteins, and DNA, is a key determinant for health promotion,

6 disease prevention, and longevity. Reactive oxygen species (ROS) have long been

7 demonstrated to play numerous roles in both physiological and pathological conditions via

8 oxidative modification of biomolecules present in cells and tissues. Accumulation of

9 oxidative damage footprints in those biological molecules has been described to be involved

10 in the onset of numerous diseases and aging [1]. In particular, biochemical modifications of

11 cellular DNA [2] and proteins [3] by ROS and lipid peroxidation products, such as acrolein

12 [4], malondialdehyde [5], and 4-hydroxy-2-nonenal [6] lead to either loss or undesired gain

13 of their functions for homeostasis disruption (Figure 1). In addition, mitochondrial DNA

14 mutations caused by ROS play significant roles in dysfunctions of cellular metabolism, and

15 defense and other systems [7], though Laqouge and Larsson have proposed that those

16 mutations may be generated by replication errors rather than accumulated oxidative damage

17 [8]. Recently, Akatsuka et al. uncovered a novel base modification, i.e.,

$181, N^{6}$-propanoadenine (acrolein-adenine), in a ferric nitrilotriacetate-treated mouse model, in 
1 which oxidative stress plays an essential role in the development of renal carcinogenesis

2 [9]. Also, mutations of the Cu,Zn-sod gene were reported to partially truncate

3 anti-oxidative systems, thereby increasing the risk of familial amyotrophic lateral sclerosis

4 (ALS) [10], a neurodegenerative disease that exhibits dysfunctions of muscle movement and

5 strength. On the other hand, proteo-stresses have been shown to be characterized to be

6 inducible by physical, chemical, and biological stimuli, and interfere with and disable

7 protein folding for denaturation [11]. Other recent studies have demonstrated that

8 accumulation of un- and mis-folded proteins may promote the formation of their aggregates,

9 the hallmark of neurodegenerative diseases, such as Alzheimer's, Parkinson's

10 and Huntington's diseases [12]. In addition, the status of cellular junk proteins is associated

11 with adiposity and thus the development of metabolic syndrome [13]. Furthermore, it should

12 be pointed out that concerted and tight regulation of the biological protein quality may

13 contribute to longevity, as demonstrated in experiments using the naked mole rats, which

14 have marked protein stability and a long life-span [14]. Thus, it is highly conceivable that

15 quality maintenance of biological proteins greatly affects health status and longevity.

16 Among a number of constitutive and inducible mechanisms that function to protect

17 biological macromolecules from oxidative stress and proteo-stress, 2 representative

18 biochemical defense systems are highlighted (Figure 2) and described below. 


\section{The Keap1/Nrf2-dependent defense system}

3

Anti-oxidation can be defined as a self-defense mechanism ubiquitously distributed

4 among organisms. Anti-oxidative modes of actions can be classified into at least the

5 following 3 categories: (1) scavenging and quenching ROS [as seen with vitamin C, vitamin

6 E, glutathione (GSH), etc.], (2) attenuation of ROS-generating enzymes (NADPH oxidase,

7 xanthine oxidase, etc.), and (3) up-regulation of anti-oxidant enzymes [superoxide dismutase

8 (SOD), catalase, etc.]. The Kelch-like ECH-associated protein 1/nuclear factor

9 (erythroid-derived 2)-like 2 (Keap1/Nrf2) system, belonging to the category (3), adaptively

10 functions to protect cells from endogenous and exogenous oxidative and electrophilic

11 damages [15] (Figure 3). In a normal state, the transcription factor Nrf2 is continuously

12 ubiquitinated by the Cul3-Keap1 ubiquitin E3 ligase complex and thereby rapidly transported

13 to degradation systems in proteasomes. ROS and electrophilic chemicals oxidize the reactive

14 cysteine residues of Keap1 in both direct and indirect manners. This critical step stabilizes

15 Nrf2, thereby inducing robust expressions of a battery of cytoprotective genes, including

16 those related to anti-oxidation [Cu/Zn-SOD, Mn-SOD, extracellular SOD,

$17 \gamma$-glutamylcysteine synthetase, etc.], xenobiotic detoxification [GSH S-transferase (GST),

18 glucuronidase, sulfatase, etc.], and protein quality control (PQC) (molecular chaperones, 
1 ubiquitin/proteasome systems, etc.) for adaptation [15]. In addition, prior to translocation of

2 Nrf2 into the nucleus, its transcription activity is modulated by several protein kinases, which

3 are simultaneously activated by stressors. Feng et al. disclosed that activation of Akt and

4 extracellular signal-regulated kinase (ERK)1/2 is required for activation of Nrf2, leading to

5 up-regulation of the expression of heme oxygenase (HO)-1, one of the major inducible

6 anti-oxidant enzymes [16]. On the other hand, Nrf2 activity is continuously repressed in a

7 normal state by Bach1, which constitutively binds to the Maf recognition element [17].

8

$9 \quad$ Heat shock proteins as molecular chaperones

Various types of stresses toward biological proteins critically disrupt their

11 conformation and folding state, which often results in abolishment of their biological

12 functions. A number of recent studies have indicated that several distinct PQC systems play

13 key roles in counteraction against proteo-stress. Heat shock proteins (HSPs), highly

14 conserved families of proteins ubiquitously expressed in most types of cells, are molecular

15 chaperones that allow misfolded and unfolded proteins to achieve functionally active

16 conformation (Figure 4). The expression and functional status of HSPs are considered to be

17 critical determinants of PQC systems and thus essentially associated with homeostasis,

18 health and longevity. In fact, expression of HSPs at high levels substantially contributes to 
1 extending the lifespan of many experimental animals [18]. HSPs are comprised of numerous

2 family proteins. Constitutive HSPs, sharing approximately $1 \%$ of cytosolic proteins, are

3 essential for maintaining PQC systems under a non-stressed condition. On the other hand,

4 various types of stressors are known to up-regulate inducible HSPs. In a normal state,

5 HSP90 , a major constitutive isoform, is bound to the transcription factor heat shock factor1

6 (Hsf1), thereby forcing it to be biologically dormant [19]. Heat shock and some other stimuli

7 are capable of dissociating this heterodimer complex. Thereafter, the resultant free Hsf1

8 forms a trimer complex and is also phosphorylated at multiple sites, finally translocating

9 into the nucleus to induce a number of HSP genes to amplify defense capacity [19]. For

10 example, HSP70, a major inducible isoform, protects neurons from protein aggregation and

11 apoptosis, which may contribute to regulate Parkinson's, Alzheimer's and polyglutamine

12 diseases, and ALS [20]. Additionally, Hsf1 has been reported to up-regulate ABC

13 transporters [21] and Bcl-2 [22], both of which potentiate cellular defense capacity against

14 foreign chemicals and other stress stimuli. Meanwhile, it is interesting to note that there are

15 mechanistic interactions between oxidative stress- and proteo-stress-triggered signaling

16 pathways. In this regard, Back et al. showed that oxidative stress chemically cleaves HSP90

17 protein, resulting in dissociation with and subsequent degradation of its client proteins,

18 including Bcr-Abl, RIP, and Akt, for cancer cell death [23]. Also, oxidative stress-triggered 
1 Keap1/Nrf2 system activation was reported to up-regulate the expression of p62, a

2 multifunctional adapter protein implicated in autophagy against proteo-stress [24]. The

3 crosstalk between oxidative stress and proteo-stress-triggered signaling pathways resembles

4 that between the complement system and toll-like receptors in innate immunity [25], and is

5 critical for first-line host defense.

6

\section{$7 \quad$ Potential side-effects of green tea polyphenols}

8 Introduction

9

Tea (Camellia sinensis, Theaceae) is a popular beverage and consumed by over

10 two-thirds of the world population [26]. Green tea polyphenols (GTPs) account for 30-42 \%

11 of the dry weight of the solids in brewed green tea, while (-)-epigallocatehin-3-gallate

12 (EGCG) (Figure 5A) is estimated to account for $50-80 \%$ of the total catechins in tea [26].

13 There is scant evidence that oral consumption of green tea, but not supplements, even at

14 large amounts has side-effects, and is actually beneficial. For example, an epidemiological

15 survey, of over 8500 people followed up for 9 years showed that the age-standardized

16 average annual incidence rate of cancer was significantly lower among females who daily

17 consumed 10 or more cups of green tea [45]. In addition to the health benefit effects of

18 GTPs [27-29], many independent studies have also suggested their harmful aspects, which 
1 are largely due to pro-oxidative properties when consumed at high doses. These

2 pro-oxidative activities are broadly attributable to their catechol structures (ortho diphenols,

3 Figure 5B). This functional moiety has a chemical characteristic to generate superoxide anion

4 radical $\left(\mathrm{O}_{2}{ }^{-}\right)$from molecular oxygen $\left({ }^{3} \mathrm{O}_{2}\right)$ through formation of an electrophilic $o$-quinone

5 counterpart. In addition, Kajiya et al. hypothesized that an ester group in EGCG plays a

6 crucial role in biochemical interactions with the lipid bilayer, providing it with a high affinity

7 to the cell membrane [30]. Of note, GTPs covalently bind protein thiols through their

8 conversion to an o-quinone counterpart [31, 32], while Elbling and colleagues showed that

9 EGCG exhibited a pro-oxidative, but not anti-oxidative, property in cellular models [33].

10 Similarly, EGCG induced injury in mouse blastocysts through intrinsic apoptotic signaling

11 processes to impair sequent embryonic development [34]. We also found that GTPs

12 increased expression of the tumor metastatic protein, pro-matrix metalloproteinase-7

13 (pro-MMP-7), in human colon adenocarcinoma cells via a pro-oxidation-dependent

14 mechanism since both SOD and catalase suppressed the production of pro-MMP-7 [35].

15 However, the pro-oxidative activity of GTPs may not be recognized as not necessarily

16 harmful because it executes induction of cancer cell apoptosis [36]. Moreover, as indicated

17 above, pro-oxidative stimuli may activate the Keap1/Nrf2 systems for exhibiting

18 anti-oxidative, anti-inflammatory, and chemopreventive activities [37]. In fact, Na et al. 
1 showed that EGCG acted as a pro-oxidant to activate Nrf2-dependent gene expressions of

2 some representative antioxidant enzymes [38, 39]. Thus, it can be argued that the

3 pro-oxidative nature of GTPs cannot be simply defined as harmful, and the risks and

4 benefits depend on the situation. Meanwhile, the anti- and pro-oxidative properties of GTPs

5 as well as many other polyphenols are determined by their doses, the presence or absence of

6 oxidants, the responsiveness of adaptation systems, and other factors.

7

8 Intestinal toxicity

9

Ulcerative colitis encompasses several chronic inflammatory disorders that cause

10 damage to the gastrointestinal tract and affect individuals of both sexes throughout life [46].

11 Dextran sulfate sodium (DSS)-induced colitis animal models are most frequently used in

12 studies of inflammatory bowel disease. Importantly, a DSS-induced model exhibited many

13 symptoms similar to those seen in human ulcerative colitis, including diarrhea, bloody feces,

14 body weight loss, mucosal ulcerations, and shortening of the colorectum [47]. We previously

15 examined the effects of the commercially available GTPs (Figure 5C) $(0.01 \%-1 \%$ in the

16 basal diet) on dimethylhydrazine (DMH)-initiated DSS-promoted mouse colorectal

17 carcinogenesis, and found that concentrations of $0.1 \%, 0.5 \%$ and $1 \%$, but not $0.01 \%$, tended

18 to increase tumor formation [48]. Furthermore, 1\% GTP treatment following the DMH 
1 exposure tended to increase histological tumor formation as compared to DMH alone group.

2 Also, the expressions of interleukin-1 $\beta$ and macrophage-migration inhibitory factor, both of

3 which play essential roles in early pro-inflammatory processes, were significantly increased

4 in the DSS + $0.5 \%$ GTP and DSS + 1\% GTP groups when compared with the DSS alone

5 group. These findings are in line with those reported by Hirose et al., who demonstrated that

6 dietary GTPs enhanced colon carcinogenesis in rats treated with DMH [49]. Moreover,

7 consumption of a $0.36 \%$ GTP diet resulted in a significant increase in multiplicity of colonic

8 tumors as compared to the carcinogen azoxymethane alone [50].

9

10 Hepatic and renal toxicity

11 A recent study found that repeated administrations of high-dose GTPs (750 mg/kg)

12 increased hepatic injuries, which were augmented under febrile conditions induced by

13 endotoxin [40]. Likewise, a single high dose of EGCG (1500 mg/kg) dramatically increased

14 plasma alanine aminotransferase (ALT) and reduced survival in male CF-1 mice [41]. In

15 addition, a green tea extract potentiated acetaminophen-induced hepatotoxicity in mice at

16 doses of 500 and $1000 \mathrm{mg} / \mathrm{kg}$ [42]. To support those experimental findings with cultured

17 cells and rodents, an epidemiological survey based on an extensive publication analysis

18 between 1999 and 2008 by Mazzanti et al. showed a causal association between green tea 
1 supplement intake and liver damage [43]. These authors speculated that this hepatotoxicity is

2 probably due to EGCG or its metabolites which can induce oxidative stress in the liver under

3 conditions related to the metabolism of patient [43]. Furthermore, Yellapu et al. implied the

4 side-effects of 'fat burners' and dietary supplements, including green tea constituents, which

5 may be hepatotoxic and may not be free of adverse effects [44]. Collectively, ingestion of

6 high doses of GTPs and other related supplements may increase the risk of hepatic injury

7 because of their pro-oxidative properties, though precise mechanisms underlying that

8 toxicity have not been fully elucidated.

9

We also assessed the potential toxicity of GTP toward liver and kidney functions

10 using a DSS-induced colitis model because, as mentioned above, high-dose GTPs may show

11 harmful effects in those organs. Importantly, oral feeding of $1 \%$ GTPs, caused kidney

12 dysfunctions in DSS-treated ICR mice, as revealed by increases in serum creatinine, the

13 most reliable biomarker of nephropathy [51]. In addition, we observed that the amounts of

14 thiobarbituric acid-reactive substances (TBARS, a potential indicator of lipid peroxidation)

15 were increased in both the livers and kidneys of the $1 \%$ GTP group. Previous

16 pharmacological studies have shown that EGCG is metabolized through methylation,

17 glucuronidation, and sulfation under normal physiological conditions, and then subsequently

18 excreted in urine [52]. It is important to note that, most, if not all, metabolites are biologically 
1 inactivated and thus much less toxic than the intact form of EGCG. Furthermore, these

2 bioactive electrophiles have a potential to bind to nucleophilic amino acid residues (e.g.,

3 cysteine) for inducing proteo-stress [53, 54], and those stress-generating properties may be

4 associated with their toxicity in the liver and kidneys. Meanwhile, in other studies high-dose

5 EGCG reduced the expressions of Nrf2-dependent antioxidant enzymes, including HO-1,

$6 \mathrm{SOD}, \mathrm{NAD}(\mathrm{P}) \mathrm{H}$ quinone oxidoreductase 1 , and catalase $[48,55]$, and some of those findings

7 showing positive correlations with our results [48, 51]. Importantly, Nrf2 activity is a

8 primary contributor to hepatoprotection [56]. Also, Lim and colleagues have found a

9 correlation between decreased SOD activity and aggravated diabetic nephropathy [57],

10 while HO-1 was reported to attenuate the progression of chronic kidney disease [58].

11 Therefore, we speculate that high-dose GTPs not only act in a pro-oxidant manner, but also

12 down-regulate Nrf2-dependent antioxidant enzymes for disordering homeostasis in the liver

13 and kidney. Downregulation of HSP70 in the colonic mucosa of DSS-exposed mice has been

14 found to have an association with intestinal inflammation [59]. It should also be noted that

15 renal and hepatic levels of HSP27 were remarkably down-regulated by $1 \%$ GTP

16 administration as compared with the DSS group [51], while more strikingly, HSP90 in the

17 kidneys and livers of the non-treated mice was notably down-regulated in mice that received

18 the $1 \%$ and even by $0.1 \%$ GTP diets. These results are consistent with those of Tran et al., 
1 who previously found that HSP90 was repressed by EGCG in MCF-7 human breast cancer

2 cells [60]. As noted above, HSP90 is the most abundant molecular chaperone and plays

3 pivotal roles in maintaining organ homeostasis [61]. Therefore, a 1\% GTP diet might induce

4 hepatic and renal toxicity by attenuating, at least in part, the expressions of anti-oxidant

5 enzymes (SOD, HO-1, etc.) and molecular chaperones (HSP90, HSP27, etc).

6

7 Beneficial effects of GTPs at low and medium doses

8

In contrast to high doses, medium- and low-dose GTP diets (0.01-0.1\%)

9 significantly inhibited rat colon carcinogenesis [62], which correlated in part with our

10 previous findings [48], i.e., a diet of $0.01 \%$, but not $0.5 \%$ or $1 \%$, GTP tended to decrease

11 tumor multiplicity or frequency. Thus, administration of GTPs exhibits both beneficial and

12 harmful effects on colon carcinogenesis, depending on the dose. In parallel, Kohlmeier et al.

13 reported that it is difficult to define the inhibitory effects of green tea consumption on colon

14 cancer development in humans [63]. Interestingly, medium and low doses of GTPs also

15 exhibited beneficial effects on hepatic and kidney functions, as GTPs at $0.01 \%$ and $0.1 \%$

16 significantly suppressed DSS-induced elevation of serum aspartate aminotransferase and

17 ALT levels [64]. Similarly, GTPs at both $0.01 \%$ and $0.1 \%$, but not $1 \%$, remarkably

18 up-regulated the expressions of HO-1 and HSP70 in livers and kidneys of DSS-exposed mice. 
1 Collectively, low- and medium-doses of GTPs have beneficial effects on DSS-induced

2 intestinal inflammation and carcinogenesis as well as hepatotoxicity and nephrotoxicity via

3 up-regulation of self-protective enzymes, while those are abolished with a high dose (Figure

$46)$.

5

6 GTPs are potential phytoalexins (plant toxins)

7

In nature, plants have a critical disadvantage for survival as compared with animals,

8 as they are unable to move to avoid biological enemies and stress stimuli, such as invading

9 microorganisms and insects, herbivorous animals, and intense sunlight. Thus, they have

10 developed specific biological systems that can adapt to and counteract against various

11 stresses. In particular, phytochemicals, biosynthesized in both constitutive and inducible

12 manners, function as a central group to fight against and mitigate exogenous stresses. This

13 raises an important question as to why phytochemicals, biosynthesized for their adaptation,

14 exhibit health benefit functions in animals, including humans. It is reasonable to speculate

15 that those chemicals have no nutritional value and are xenobiotics substances in animals.

16 Although mammals efficiently and actively absorb and utilize primary products, including

17 sugars, protein, and lipids, as essential nutrients, the bioavailability of phytochemicals in

18 experimental rodents and humans has been consistently reported to be fairly poor in several 
1 studies. For example, a bioavailability study showed that administration of EGCG resulted

2 in its substantial biotransformation, e.g., glucuronidation, sulfation, and $O$-methylation, and

3 its blood concentrations were quite limited [65, 66]. Therefore, most, if not all,

4 phytochemicals are substantially foreign chemicals to mammals, and it is not surprising that

5 they are actively subjected to detoxification and excretion systems. Based on those findings, it

6 is reasonable that phytochemicals up-regulate anti-oxidant enzymes, xenobiotic

7 detoxification proteins, and molecular chaperones, all of which are recognized as defense

8 molecules against oxidative- and proteo-stresses induced by those chemicals. Of paramount

9 importance, some of those adaptive responses have mechanistic links to their already-known,

10 beneficial bioactivities $[67,68]$. Exposure levels to those foreign chemicals must essentially

11 remain at mild ranges to gain beneficial effects, otherwise, no positive effects or catastrophic

12 outcomes would become dominant. This notion is in line with the concept of hormesis

13 [69-71], in which mild biological or physical stresses at mild levels activate defense systems

14 for adaptation (Figure 7).

15

\section{Conclusion} High doses, but not low and medium doses, of dietary GTPs deteriorated colitis and

18 colorectal cancer, and also induced liver and kidney dysfunctions in mice. The fundamental 
1 reason why phytochemicals trigger activation of self-defense systems is probably because

2 they are essentially foreign chemicals with no nutritional value mammals. Accumulated

3 evidence regarding the functionality and toxicity of phytochemicals reminds us of the saying

4 'More than enough is too much'.

5

\section{Acknowledgements}

7

The author wishes to thank Drs. Mihye Kim and Hirofumi Inoue, as well as Dr.

8 Takuji Tanaka (Tohkai Cytopathology Institute, Gifu, Japan) for their great contributions to

9 our studies cited in this manuscript. This work was supported by Research and Development

10 Projects for Application in Promoting New Policy of Agriculture Forestry and Fisheries, from

11 the Ministry of Agriculture, Forestry and Fisheries, Japan (Grant No. 23005).

12

13 Disclosure

14 There are no financial or contractual agreements that might cause conflicts of interest

15 or be perceived as causing conflicts of interest in regard to this study.

16

17 References

18 [1] S.I. Liochev, Free Radic Biol Med 60 (2013) 1-4.

19 [2] K.S. Gates, Chem Res Toxicol 22 (2009) 1747-1760. 
1 [3] P.A. Grimsrud, H. Xie, T.J. Griffin, D.A. Bernlohr, J Biol Chem 283 (2008) 21837-21841.

[4] M. Singh, T.N. Dang, M. Arseneault, C. Ramassamy, J Alzheimers Dis 21 (2010) 741-756.

5] R. Sultana, M. Perluigi, D. Allan Butterfield, Free Radic Biol Med 62 (2013) 157-169.

[6] M. Perluigi, R. Coccia, D.A. Butterfield, Antioxid Redox Signal 17 (2012) 1590-1609.

[7] C.H. Wang, S.B. Wu, Y.T. Wu, Y.H. Wei, Exp Biol Med (Maywood) 238 (2013) 450-460.

[8] M. Lagouge, N.G. Larsson, J Intern Med 273 (2013) 529-543.

[9] S. Akatsuka, T.T. Aung, K.K. Dutta, L. Jiang, W.H. Lee, Y.T. Liu, J. Onuki, T. Shirase, K. Yamasaki, H. Ochi, Y. Naito, T. Yoshikawa, H. Kasai, Y. Tominaga, K. Sakumi, Y. Nakabeppu, Y. Kawai, K. Uchida, A. Yamasaki, T. Tsuruyama, Y. Yamada, S. Toyokuni, Am J Pathol 169 (2006) 1328-1342.

[10] M.R. Cookson, F.M. Menzies, P. Manning, C.J. Eggett, D.A. Figlewicz, C.J. McNeil, P.J. Shaw, Amyotroph Lateral Scler Other Motor Neuron Disord 3 (2002) 75-85.

[11] H.J. Kim, N.R. Hwang, K.J. Lee, Mol Cells 23 (2007) 123-131.

[12] A. Naeem, N.A. Fazili, Cell Biochem Biophys 61 (2011) 237-250.

[13] P. Christian, J. Sacco, K. Adeli, Biochim Biophys Acta 1831 (2013) 819-824.

[14] V.I. Pérez, R. Buffenstein, V. Masamsetti, S. Leonard, A.B. Salmon, J. Mele, B. Andziak, T. Yang, Y. Edrey, B. Friguet, W. Ward, A. Richardson, A. Chaudhuri, Proc Natl Acad Sci U S A 106 (2009) 3059-3064.

[15] M. Kobayashi, M. Yamamoto, Antioxid Redox Signal 7 (2005) 385-394.

[16] J. Feng, P. Zhang, X. Chen, G. He, J Cell Biochem 112 (2011) 1524-1531.

[17] J. Sun, M. Brand, Y. Zenke, S. Tashiro, M. Groudine, K. Igarashi, Proc Natl Acad Sci U S A 101 (2004) 1461-1466.

[18] S.K. Calderwood, A. Murshid, T. Prince, Gerontology 55 (2009) 550-558.

[19] E.S. Christians, L.J. Yan, I.J. Benjamin, Crit Care Med 30 (2002) S43-50.

[20] G. Turturici, G. Sconzo, F. Geraci, Biochem Res Int 2011 (2011) 618127.

[21] N. Vydra, A. Toma, M. Glowala-Kosinska, A. Gogler-Piglowska, W. Widlak, BMC Cancer 13 (2013) 504.

[22] A.T. Jacobs, L.J. Marnett, J Biol Chem 282 (2007) 33412-33420.

[23] R. Beck, J. Verrax, T. Gonze, M. Zappone, R.C. Pedrosa, H. Taper, O. Feron, P.B. Calderon, Biochem Pharmacol 77 (2009) 375-383.

[24] I.P. Nezis, H. Stenmark, Antioxid Redox Signal 17 (2012) 786-793. 
1 [25] W.C. Song, Toxicol Pathol 40 (2012) 174-182.

2 [26] D.A. Balentine, S.A. Wiseman, L.C. Bouwens, Crit Rev Food Sci Nutr 37 (1997)

3

4 [27] N. Thavanesan, Br J Nutr 106 (2011) 1297-1309.

5

6

7

8

9

10

11

12

13

14

15

16

17

18

19

20

21

22

23

24

25

26

27

28

29

30

31

32

33

34

35

36 693-704.

[28] C.S. Yang, H. Wang, Mol Nutr Food Res 55 (2011) 819-831. 187-208. 2638-2643. Biochem 74 (2010) 2451-2456.

[34] Y.C. Fan, W.H. Chan, Drug Chem Toxicol (2013). 1553-1562. (2003) 1369-1378. Biophys 476 (2008) 171-177.

[39] H.K. Na, Y.J. Surh, Food Chem Toxicol 46 (2008) 1271-1278. I.A. Khan, M.K. Ashfaq, Fitoterapia 90 (2013) 151-159. Toxicol 48 (2010) 409-416. Toxicol 50 (2012) 1439-1446. S. Mastrangelo, Eur J Clin Pharmacol 65 (2009) 331-341. (2011) 157-160.

[45] K. Imai, K. Suga, K. Nakachi, Prev Med 26 (1997) 769-775.

[29] S.A. Mandel, T. Amit, O. Weinreb, M.B. Youdim, J Alzheimers Dis 25 (2011)

[30] K. Kajiya, S. Kumazawa, T. Nakayama, Biosci Biotechnol Biochem 65 (2001)

[31] T. Mori, T. Ishii, M. Akagawa, Y. Nakamura, T. Nakayama, Biosci Biotechnol

[32] T. Ishii, T. Ichikawa, K. Minoda, K. Kusaka, S. Ito, Y. Suzuki, M. Akagawa, K. Mochizuki, T. Goda, T. Nakayama, Biosci Biotechnol Biochem 75 (2011) 100-106.

[33] L. Elbling, R.M. Weiss, O. Teufelhofer, M. Uhl, S. Knasmueller, R. Schulte-Hermann, W. Berger, M. Micksche, FASEB J 19 (2005) 807-809.

[35] M. Kim, A. Murakami, K. Kawabata, H. Ohigashi, Carcinogenesis 26 (2005)

[36] C. Chen, G. Shen, V. Hebbar, R. Hu, E.D. Owuor, A.N. Kong, Carcinogenesis 24

[37] J.D. Lambert, R.J. Elias, Arch Biochem Biophys 501 (2010) 65-72.

[38] H.K. Na, E.H. Kim, J.H. Jung, H.H. Lee, J.W. Hyun, Y.J. Surh, Arch Biochem

[40] I.G. Saleh, Z. Ali, N. Abe, F.D. Wilson, F.M. Hamada, M.F. Abd-Ellah, L.A. Walker,

[41] J.D. Lambert, M.J. Kennett, S. Sang, K.R. Reuhl, J. Ju, C.S. Yang, Food Chem

[42] W.F. Salminen, X. Yang, Q. Shi, J. Greenhaw, K. Davis, A.A. Ali, Food Chem

[43] G. Mazzanti, F. Menniti-Ippolito, P.A. Moro, F. Cassetti, R. Raschetti, C. Santuccio,

[44] R.K. Yellapu, V. Mittal, P. Grewal, M. Fiel, T. Schiano, Can J Gastroenterol 25

[46] W. Strober, B.R. Lúdvíksson, I.J. Fuss, Ann Intern Med 128 (1998) 848-856. 
1 [47] I. Okayasu, S. Hatakeyama, M. Yamada, T. Ohkusa, Y. Inagaki, R. Nakaya, Gastroenterology 98 (1990) 694-702.

[48] M. Kim, A. Murakami, S. Miyamoto, T. Tanaka, H. Ohigashi, Biofactors 36 (2010) 43-51.

[49] M. Hirose, T. Hoshiya, Y. Mizoguchi, A. Nakamura, K. Akagi, T. Shirai, Cancer Lett 168 (2001) 23-29.

[50] J.H. Weisburger, A. Rivenson, J. Reinhardt, C. Aliaga, J. Braley, B. Pittman, E. Zang, Carcinogenesis 19 (1998) 229-232.

[51] H. Inoue, S. Akiyama, M. Maeda-Yamamoto, A. Nesumi, T. Tanaka, A. Murakami, Cell Stress Chaperones 16 (2011) 653-662.

[52] K. Okushio, M. Suzuki, N. Matsumoto, F. Nanjo, Y. Hara, Biosci Biotechnol Biochem 63 (1999) 430-432.

[53] K. Ohnishi, E. Nakahata, K. Irie, A. Murakami, Biochem Biophys Res Commun 430 (2013) 616-622.

[54] K. Ohnishi, S. Ohkura, E. Nakahata, A. Ishisaka, Y. Kawai, J. Terao, T. Mori, T. Ishii, T. Nakayama, N. Kioka, S. Matsumoto, Y. Ikeda, M. Akiyama, K. Irie, A. Murakami, PLoS One 8 (2013) e58641.

[55] M.H. Kweon, V.M. Adhami, J.S. Lee, H. Mukhtar, J Biol Chem 281 (2006) 33761-33772.

[56] A.D. Patterson, B.A. Carlson, F. Li, J.A. Bonzo, M.H. Yoo, K.W. Krausz, M. Conrad, C. Chen, F.J. Gonzalez, D.L. Hatfield, Chem Res Toxicol (2013).

[57] J.H. Lim, D.Y. Youn, H.J. Yoo, H.H. Yoon, M.Y. Kim, S. Chung, Y.S. Kim, Y.S. Chang, C.W. Park, J.H. Lee, Diabetologia (2013).

[58] N. Desbuards, J.M. Hyvelin, M.C. Machet, V. Eder, M.A. Garrigue, J.M. Halimi, D. Antier, Nephron Exp Nephrol 113 (2009) e35-44.

[59] S. Bhattacharyya, P.K. Dudeja, J.K. Tobacman, Inflamm Bowel Dis 15 (2009) 673-683.

[60] Y. Li, T. Zhang, Y. Jiang, H.F. Lee, S.J. Schwartz, D. Sun, Mol Pharm 6 (2009) 1152-1159.

[61] Q. Shi, Z. Dong, H. Wei, Cell Mol Immunol 4 (2007) 53-57.

[62] T. Yamane, N. Hagiwara, M. Tateishi, S. Akachi, M. Kim, J. Okuzumi, Y. Kitao, M. Inagake, K. Kuwata, T. Takahashi, Jpn J Cancer Res 82 (1991) 1336-1339.

[63] L. Kohlmeier, K.G. Weterings, S. Steck, F.J. Kok, Nutr Cancer 27 (1997) 1-13.

[64] H. Inoue, M. Maeda-Yamamoto, A. Nesumi, T. Tanaka, A. Murakami, Biosci Biotechnol Biochem 77 (2013) 1223-1228.

[65] S.B. Moyers, N.B. Kumar, Nutr Rev 62 (2004) 204-211. 
1 [66] M.J. Lee, P. Maliakal, L. Chen, X. Meng, F.Y. Bondoc, S. Prabhu, G. Lambert, S.

2

3

4

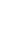

\section{6}

\section{7}

\section{8}

9

10

11

12

Figure legends

13

14

Modifications of cellular membrane lipids, proteins, and DNA by reactive oxygen species and electrophilic aldehyde compounds, the latter of which are degradation products of lipid

16 peroxide. ROS, reactive oxygen species; ACR, acrolein; MDA, malondialdehyde; HNE, 4-hydroxy-2-nonenal; HS, heat shock

18

19 Figure 2

20 Adaptive defense systems for oxidative stress and proteo-stress, which are specifically

21 sensed by Keap1 and HSP90 $\beta$, respectively, for activating the key transcription factors, Nrf2

22 and HSF1 to induce robust expressions of numerous genes for adaptation. Nrf2, nuclear 
1 GST, glutathione S-transferase; NQO1, NAD(P)H dehydrogenase 1; HSP, heat shock

2 protein; HSF1, heat shock factor1; ABC, ATP-binding cassette

3

\section{$4 \quad$ Figure 3}

5 Mechanistic scheme for Nrf2 activation and resultant expressions of self-defense genes. Ub,

6 ubiquitin; ERK, extracellular signal-related kinase

7

\section{Figure 4}

9 Proteo-stress-induced HSF1-dependent activation of HSP40 and HSP70 protein expressions

10 for increased proteo-stress resistance. HSP, heat shock protein; Hsf1, heat shock factor1;

11 HSE, heat shock element

12

$13 \quad$ Figure 5

14 (A) Chemical structure of EGCG, (B) formation of o-quinone counterpart and its

15 reaction with protein thiols to form a covalent bond, (C) composition of GTPs used in our

16 experiments

17

18 Figure 6 
1 Summary of results of experiments on effects of low medium, and high doses of GTPs on

2 large intestine, liver, and kidneys of ICR mice. Summarized from ref. [50] and [63]. TRABS,

3 thiobarbituric acid reactive substances; AST, aspartate aminotransferase; ALT, alanine

4 aminotransferase; HSP, heat shock protein; HO-1, heme oxygenase-1

5

\section{$6 \quad$ Figure 7}

7 Hormetic curve illustrating the relationship between the stress intensity (dose) and

8 homeostatic capacity. The hormetic zone areas differ among the various stressors, with a

9 more frequent chance of adaptation with a larger zone 


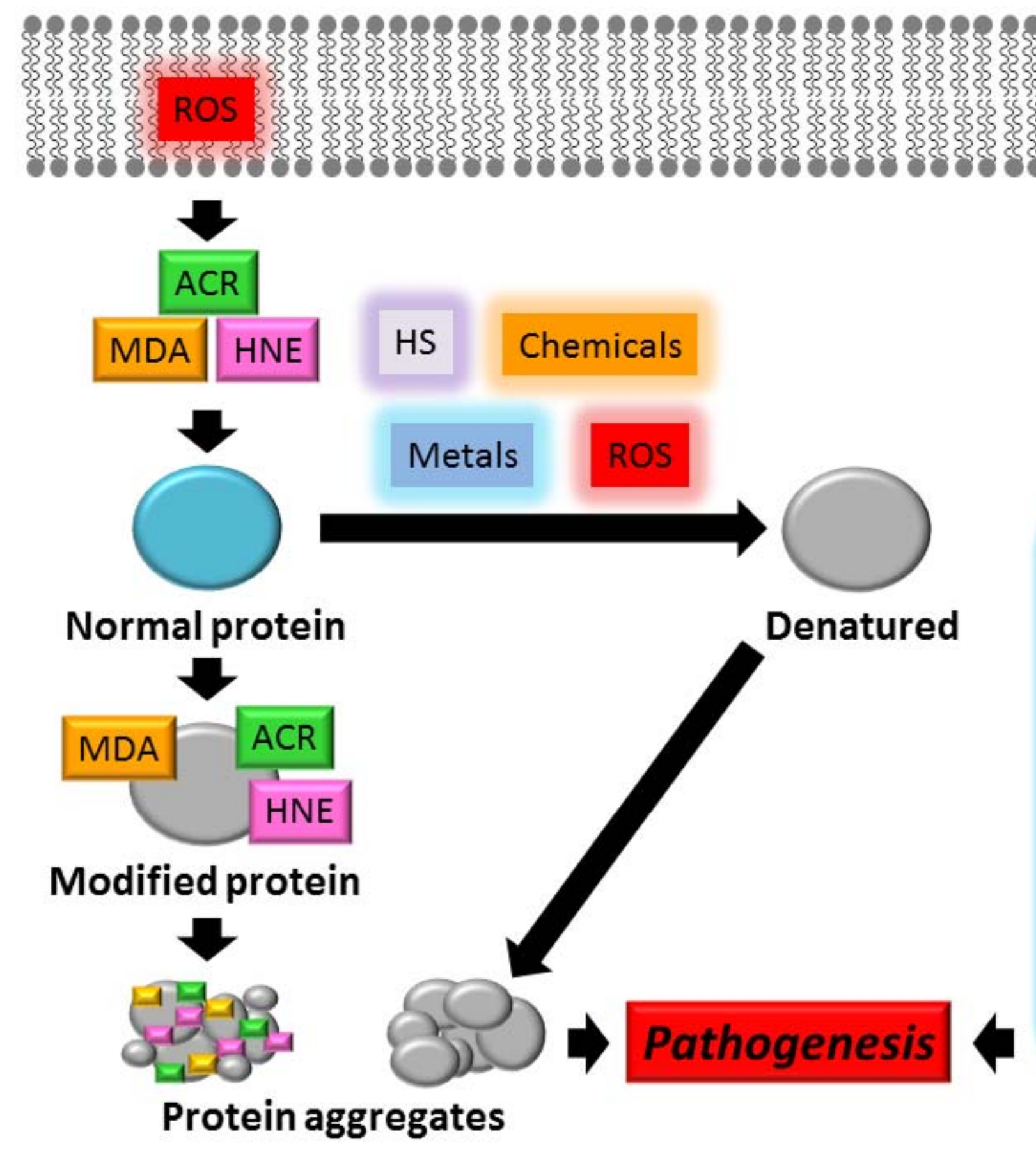

Cellular membrane

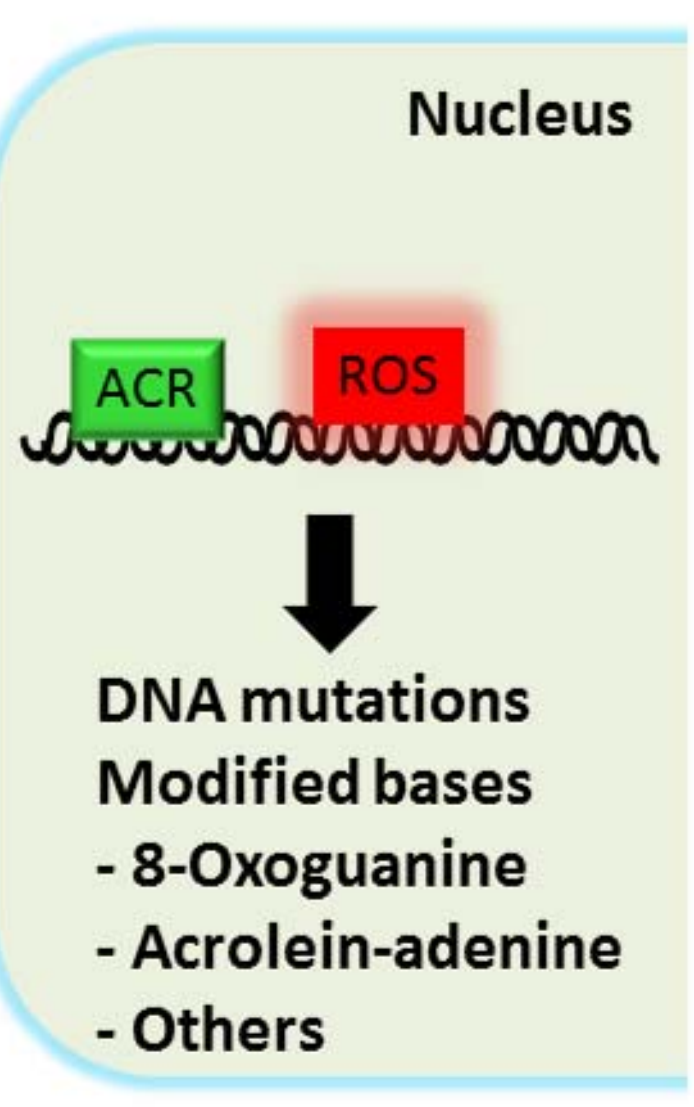

Figure 1 


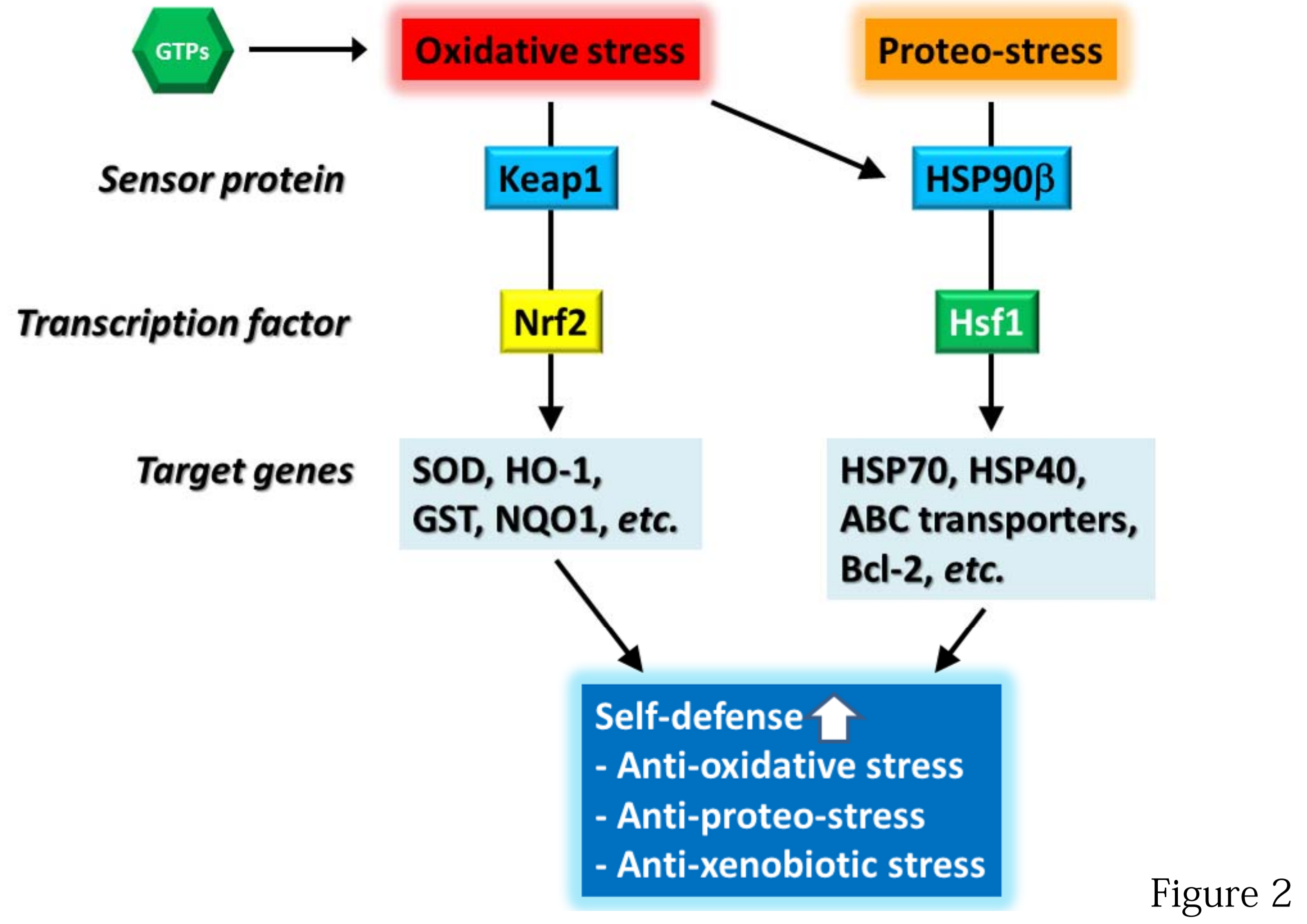




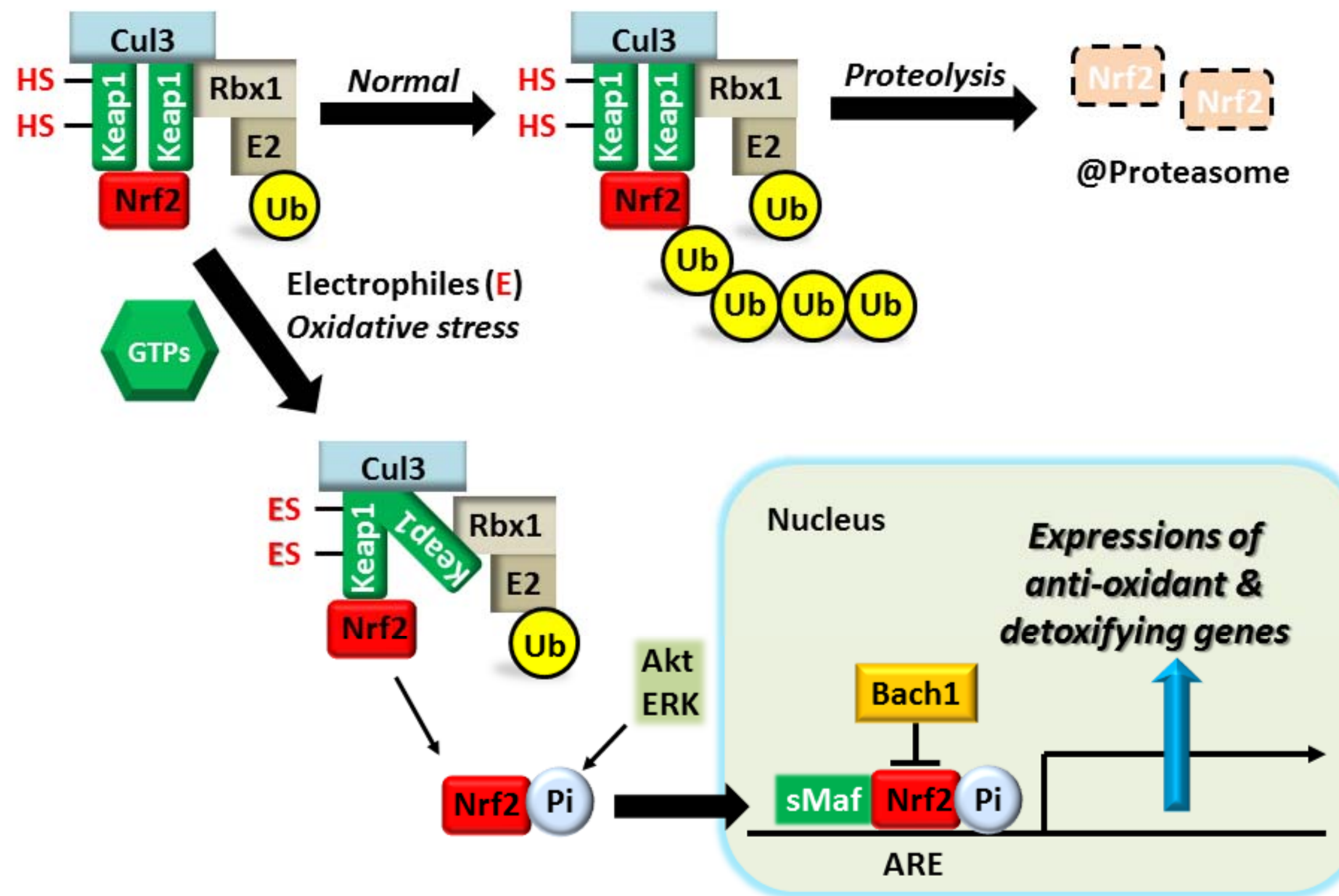

Figure 3 

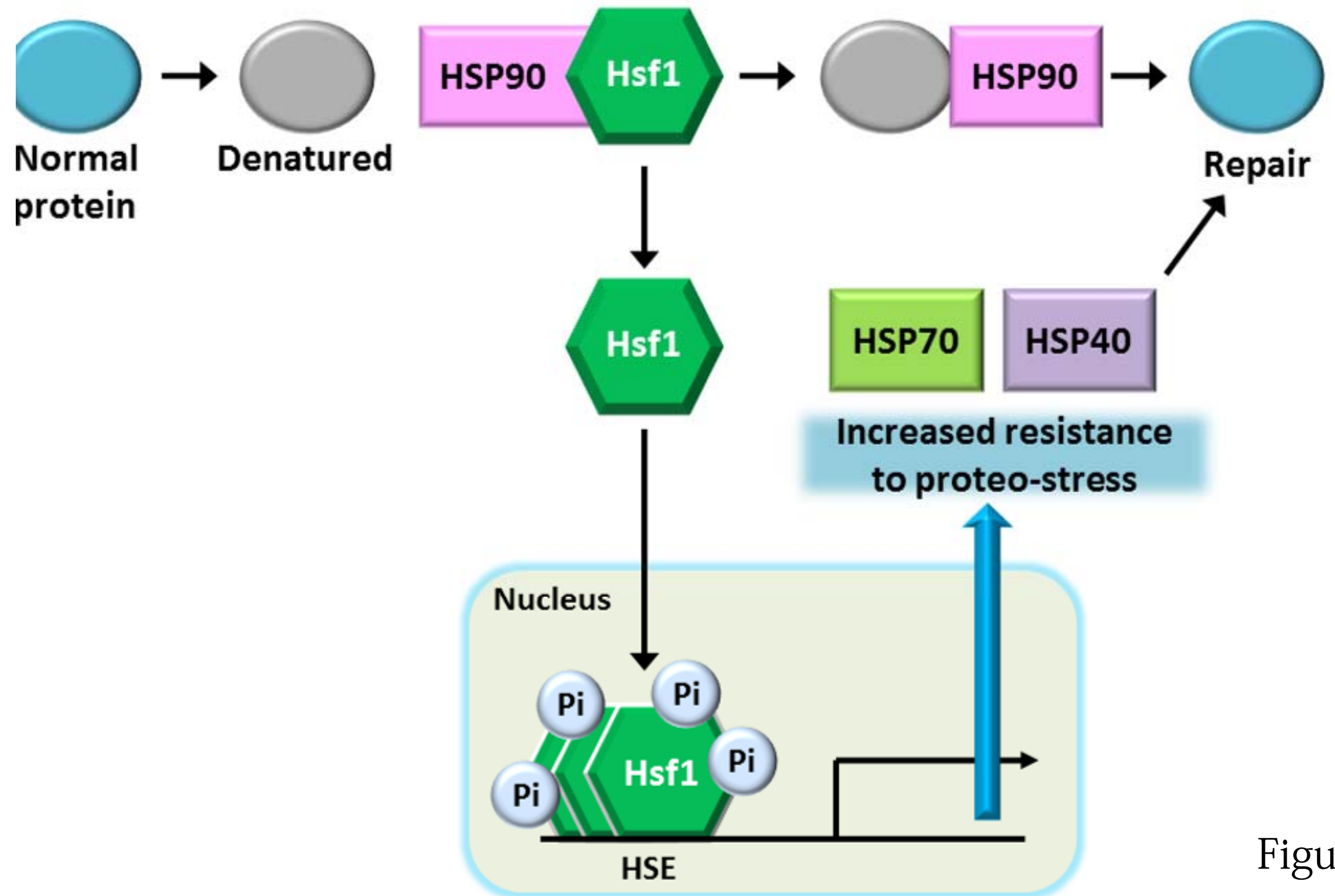

Figure 4 
A

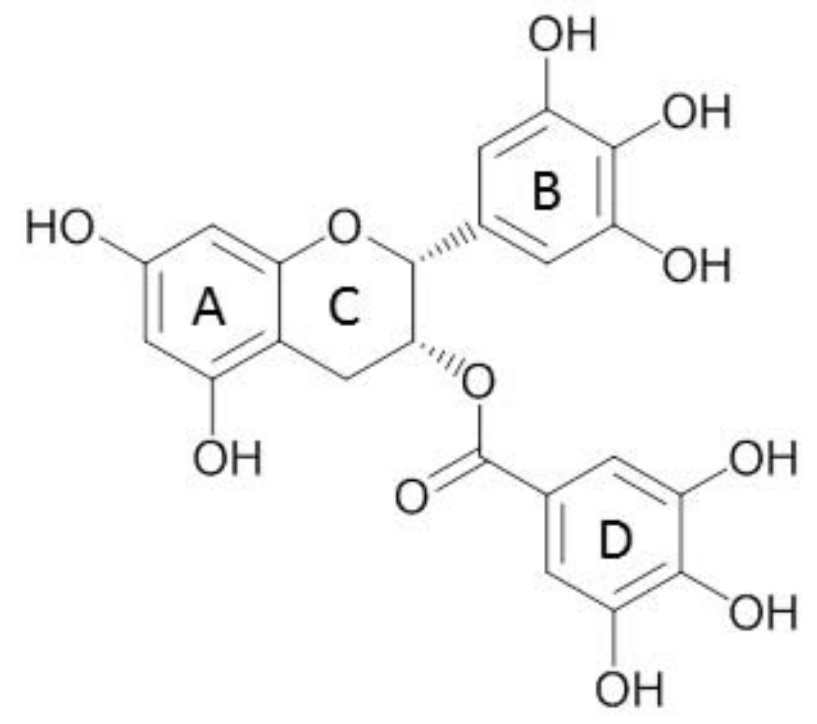

C

\begin{tabular}{|l|c|}
\hline Component & $\%$ \\
\hline Polyphenols & 95 \\
\hline Catechins & 70 \\
\hline EGCG & 35 \\
\hline Caffeine & 3 \\
\hline Water & 5 \\
\hline
\end{tabular}

Figure 5 
Normal intestine

\section{\begin{tabular}{|l|l|}
\hline $0.01 \%$ & $0.1 \%$
\end{tabular}}

Survival

rate

Kidney wt.

Liver wt.

Spleen wt.

TBARS

AST/ALT

Creatinine

HSPs, HO-1,

etc.

Ameliorated
Inflamed intestine

\begin{tabular}{|l|l|l|}
\hline $0.01 \%$ & $0.1 \%$ & $1 \%$ \\
\hline
\end{tabular}

$1 \%$

$0.01 \%$
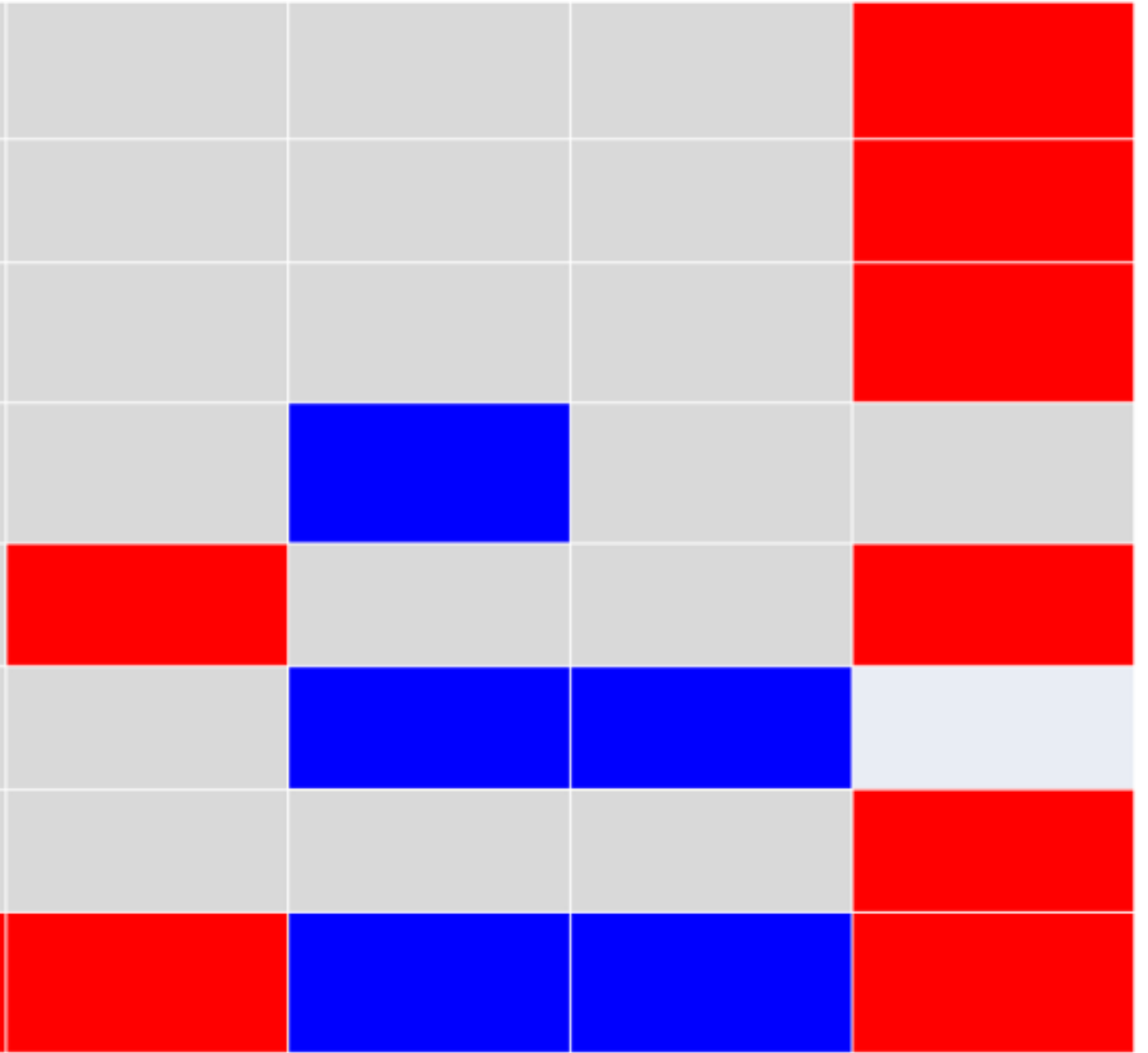

No significant change 


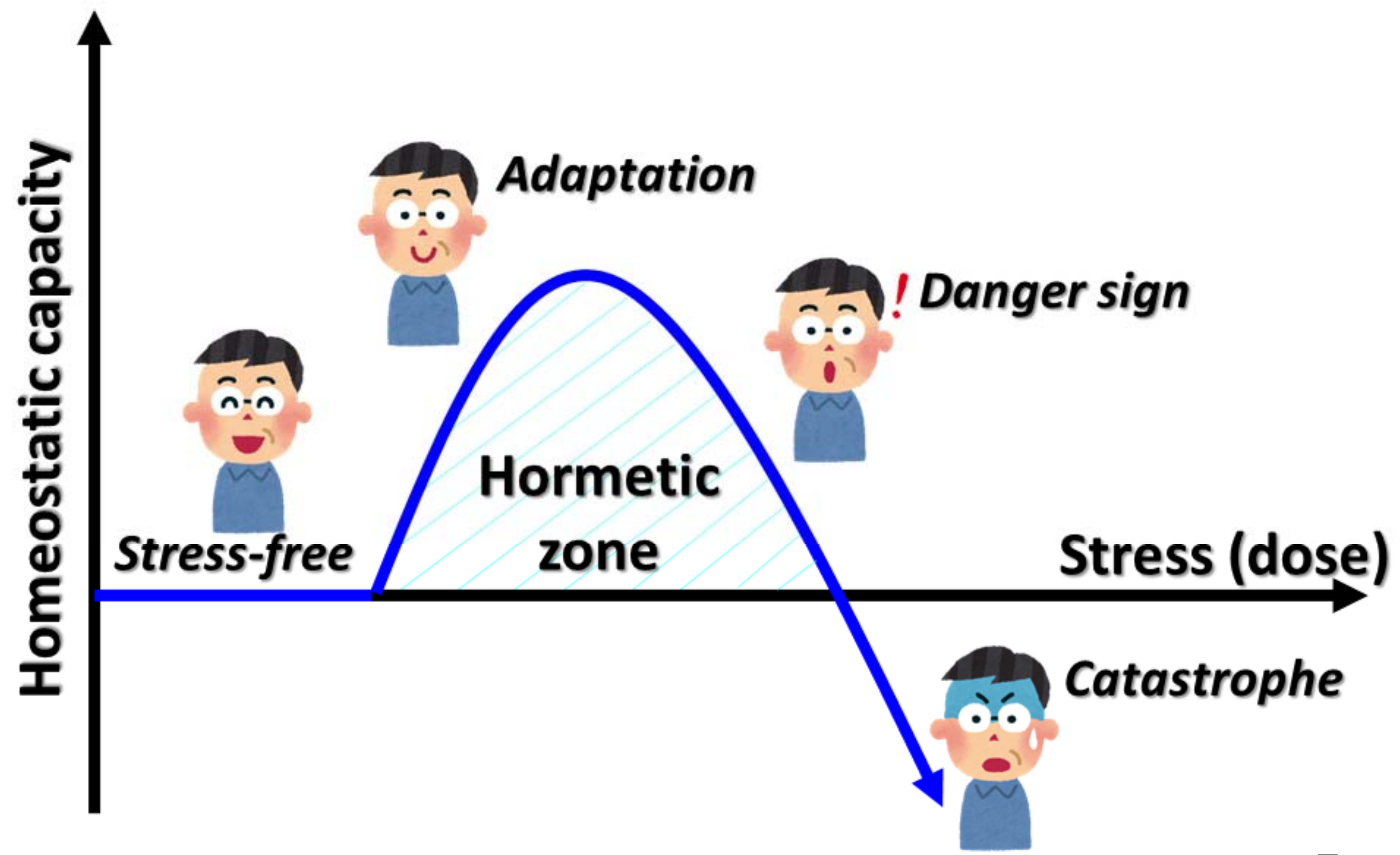

Figure 7 